\section{Versatile Constant Transmission Photochemical Reactor}

THE ultra-violet irradiation of organic and organometallic compounds in the liquid phase is often complicated by the tendency for deposition of an opaque layer of polymeric materials and other reaction products on the cell walls. With the use of apparatus of conventional type this tendency can cause the observed rates of photochemical reactions to decrease rapidly with time, and thereby make it impossible to investigate some reactions beyond very low conversions of the starting materials. Accurate quantitative work on relative reaction rates and quantum yields becomes very difficult.

We now describe a photochemical reactor in which the transmission of the cell can be kept constant over long periods even under irradiation conditions where an inner t,ransmitting surface would normally be rapidly occluded. This reactor is illustrated in Fig. 1 and has as its key feature an arrangement whereby the inner surface in contact with the photolysate is continuously rubbed by means of a mechanically rotated pad of fused silica fibre. Temperature control is achieved by the circulation of water through an outer jacket of fused silica and, if necessary, a thermostat device of conventional type. Various optical filter solu. tions can be used in place of water when it is desired to isolate a particular wavelength range. Under normal conditions of use, and with occasional reversal of the direction of rotation of the scrubber pad, the transmission of the cell remains effectively constant for periods of $20-40 \mathrm{~h}$ or even longer. Ordinary glass fibre has been used in place of silica fibre, but is slightly less durable. Any conventional external ultra-violet radiation source can be used, preferably with an aluminium reflector. We use up to three 500 -W medium pressure Hanovia lamps for routine purposes. The design is an improved version of one previously reported from this laboratory ${ }^{1}$.

The following typical result illustrates the general utility. Irradiation of a solution of cyclooctene in benzene under nitrogen is known to produce a $1: 1$ adduct of structure $(I)^{2}$. If the pad of fused silica fibre is not rotated,

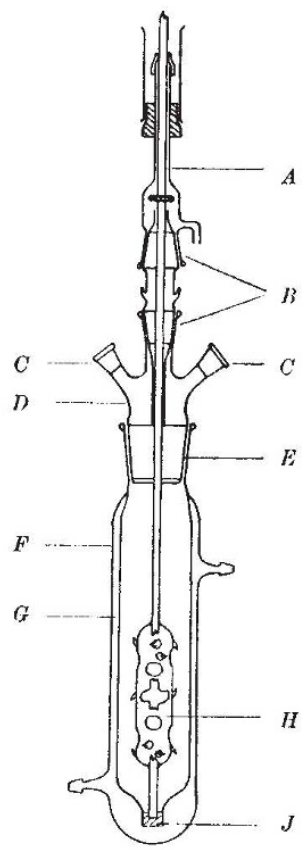
Fig. 1. $A$, Stirrer guide designed to avoid contamination with Nujol
lubricant (ref. 3 ); $B$, ' $B$ 19' joints; $C$, ' $B 14$ ' joints; $D$, borosilicate glass; lubricant (ref. 3 ); $B$, ' $B 19$ ' joints; $C$, ' $B$ 14' joints; $D$, borosilicate glass $E, \quad B 50$ ' joint with 'Teflon' sleeve; $F$, fused silica cell; overall length $35 \mathrm{~cm}$, outside diameter $8 \mathrm{~cm}$, working capacity about $400 \mathrm{ml}$.; $G$, annular gap of $1 \mathrm{~cm}$ or more for filter solution/coolant; $\boldsymbol{H}$, bollow perforated borosilicate glass cylinder with hooks for attachment of fused (the relative length can be greater than that
$J$, bearing of 'Teflon' reinforced with glass flbre.

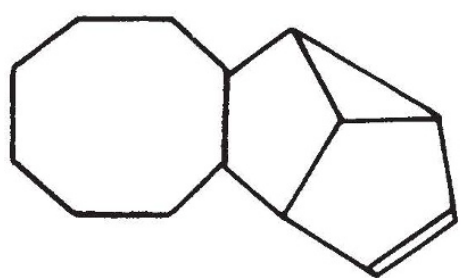

(I)

the rate of formation of adduct (I) falls rapidly with time from the initial value (about $2 \mathrm{~g} / \mathrm{h}$ with one 500 -W medium pressure Hanovia lamp) and is virtually zero within about $9 \mathrm{~h}$. Under similar conditions, but with operation of the scrubber as described above, the initial rate of formation of adduct $(\mathrm{I})$ is readily maintained for over $24 \mathrm{~h}$.

The constant-transmission principle of this reactor has an obvious extension to gas-phase investigations.

D. BRYCE-SMith

J. A. Frost

A. Grlbert

Department of Chemistry,

University of Reading.

Whiteknights Park,

Reading.

Received February 14, 1967.

1 Blair, J. M., Bryce-Smith, D., and Pengilly, B. W., J. Chem. Soc., 3174 (1959). ${ }^{2}$ Bryce-Smith, D., Gilbert, A., and Orger, B. H., Chem. Commun., 512 (1966). ${ }^{3}$ Connett, B. E., and Frost, J. A., Lab. Practice, 85 (1966).

\section{Spin Polarization in Atoms and $\pi$-Radicals}

IN certain radicals, sometimes referred to as $\sigma$-radicals ${ }^{1,2}$, the contribution to the orbital of the unpaired electron from one or more of the atoms involved includes a relatively high atomic 8 character, and this is manifested as a large isotropic hyperfine coupling. It is customary to estimate the $s$ character by dividing this isotropic coupling by a calculated value $\left(A^{\theta}\right)$ for a spin density of unity in the appropriate orbital ${ }^{2}$. For $\pi$-radicals the isotropic hyperfine coupling constants of which are a consequence of spin-polarization or configuration interaction, however, the value is generally related, not to the atomic value, $A^{0}$, but to the value $(Q)$ that would have been obtained had the spin density in the $p$ orbital of the atom concerned been unity.

Two correlations are revealed if these $Q$ values are divided by the appropriate $A^{0}$ value. (We have suggested that this ratio be given the symbol $U$.) The first is that when this is done for atoms such as nitrogen (normalized to one electron), the result is close to $0 \cdot 2$ per cent. The second is that for molecules having three $\sigma$-bonds to the atom in question the result is close to 4 per cent, and for all radicals tested it is never less than about 2 per cent. Although results for particular atoms and molecules have been discussed in great detail and refinement, this remarkable lack of sensitivity of the $U$ values to the nature of the nucleus and bonding does not seem to have been recognized. Even more striking is the very large difference between results for atoms and those for molecules. This suggests to us that the dominating factor for molecules is polarization of the $\sigma$-bonding electrons and to a less extent non-bonding valence electrons having $p-s$ hybrid character, rather than of the core $s$-electrons. Perhaps the significant difference is the fact that for the molecules, the effective electrons are greatly attenuated away from the nucleus concerned and that this magnifies any spin-imbalance at this nucleus.

Details of these results will be published elsewhere. Here we wish to draw attention to the considerable utility of $U$ values for radicals especially when considering the possibility of minor deviations from planarity ${ }^{3}$. 